\title{
A DUALITY PROOF OF A THEOREM OF P. HILL
}

\author{
RANDALL R. HOLMES
}

(Communicated by Ronald Solomon)

\begin{abstract}
A duality argument is given to prove the equivalence of a recent theorem of P. Hill and the main step in Zippin's proof of Ulm's theorem.
\end{abstract}

Let $G$ and $H$ be isomorphic finite abelian $p$-groups with isomorphic subgroups $A$ and $B$, respectively, and set $X=G / A, Y=H / B$. In his 1935 proof of Ulm's theorem, Zippin established the following result.

Theorem (Zippin [2, p. 30], [3]). If $\varphi: A \rightarrow B$ is an isomorphism, then there exists an isomorphism $G \rightarrow H$ that induces $\varphi$ if and only if $\varphi$ preserves heights.

Recently, P. Hill has proved the following theorem (stated in a slightly different but equivalent form).

Theorem (P. Hill [1]). If $\psi: X \rightarrow Y$ is an isomorphism, then there exists an isomorphism $G \rightarrow H$ that induces $\psi$ if and only if $\psi$ preserves coset covalues.

Hill's theorem appears to be dual to Zippin's theorem. The purpose of this note is to make the connection between these two results more explicit by giving a duality proof of their equivalence.

First, recall the definitions. Let $P$ be any finite abelian $p$-group and let $0 \neq x \in P$. The height of $x$ in $P$ is the unique integer $k \geq 0$ for which $x \in p^{k} P \backslash p^{k+1} P$ (and the height of 0 is $\infty$, by convention). Similarly, the exponent of $x$ in $P$ is the unique integer $k>0$ for which $x \in P\left[p^{k}\right] \backslash P\left[p^{k-1}\right]$ where $P\left[p^{k}\right]:=\left\{y \in P \mid p^{k} y=0\right\}$ (and the exponent of 0 is zero). If $S \leq P$, then the coset covalue of $x+S$ is by definition the least among the exponents of the elements of $x+S$.

With the notation as above, set $A_{k}=A \cap p^{k} G$ and $X^{k}=G /\left(A+G\left[p^{k}\right]\right)$, and define $B_{k}$ and $Y^{k}$ similarly. Let $\pi_{X}: G \rightarrow X$ and $\pi_{Y}: G \rightarrow Y$ denote the canonical epimorphisms.

Lemma. (i) An isomorphism $A \rightarrow B$ preserves heights if and only if it induces an isomorphism $A_{k} \rightarrow B_{k}$ for each $k$.

(ii) An isomorphism $X \rightarrow Y$ preserves coset covalues if and only if it induces an isomorphism $X^{k} \rightarrow Y^{k}$ for each $k$.

Proof. The proof of (i) is trivial.

Received by the editors May 5, 1993.

1991 Mathematics Subject Classification. Primary 20K30; Secondary 20 E07. 
(ii) We can make the identifications

$$
X^{k}=X / \pi_{X}\left(G\left[p^{k}\right]\right) \quad \text { and } \quad Y^{k}=Y / \pi_{Y}\left(H\left[p^{k}\right]\right)
$$

so that an isomorphism $X \rightarrow Y$ induces an isomorphism $X^{k} \rightarrow Y^{k}$ if and only if it induces an isomorphism $\pi_{X}\left(G\left[p^{k}\right]\right) \rightarrow \pi_{Y}\left(H\left[p^{k}\right]\right)$. The statement now follows since clearly the coset covalue of a coset $g+A$ is $k$ if and only if $g+A \in \pi_{X}\left(G\left[p^{k}\right]\right) \backslash \pi_{X}\left(G\left[p^{k-1}\right]\right)$.

In view of this lemma, the equivalence of Zippin's theorem and Hill's theorem can be stated as follows.

Theorem. The following statements are equivalent.

(Z) If $\varphi: A \rightarrow B$ is an isomorphism, then there exists an isomorphism $G \rightarrow$ $H$ that induces $\varphi$ if and only if $\varphi$ induces an isomorphism $A_{k} \rightarrow B_{k}$ for each $k$.

(H) If $\psi: X \rightarrow Y$ is an isomorphism, then there exists an isomorphism $G \rightarrow$ $H$ that induces $\psi$ if and only if $\psi$ induces an isomorphism $X^{k} \rightarrow Y^{k}$ for each $k$.

We need some preliminaries before proving the theorem. Denote the contravariant functor $\operatorname{Hom}\left(\cdot, \mathbb{Z}\left(p^{\infty}\right)\right)$ on finite abelian $p$-groups by $P \mapsto P^{*}$ (object map) and $\varphi \mapsto \varphi^{*}$ (morphism map). This functor is exact and $P^{* *}$ is naturally isomorphic to $P$ (cf. [2, pp. 79-80]). As usual, if $S \leq P$, we identify $S^{*}$ with $P^{*} / S^{0}$ and $(P / S)^{*}$ with $S^{0}$ where $S^{0}=\left\{f \in P^{*} \mid f(S)=0\right\}$.

Lemma 1. Let $\eta: G \rightarrow H$ be a homomorphism.

(i) If $\eta$ induces a homomorphism $\psi: X \rightarrow Y$, then $\eta^{*}$ induces $\psi^{*}$.

(ii) If $\eta$ induces a homomorphism $\varphi: A \rightarrow B$, then $\eta^{*}$ induces $\varphi^{*}$.

Proof. (i) Assume $\eta$ induces a homomorphism $\psi: X \rightarrow Y$. This means that $\psi \circ \pi_{X}=\pi_{Y} \circ \eta$. Applying $*$, we have $\pi_{X}^{*} \circ \psi^{*}=\eta^{*} \circ \pi_{Y}^{*}$. Since $\pi_{X}^{*}: X^{*} \rightarrow G^{*}$ and $\pi_{Y}^{*}: Y^{*} \rightarrow H^{*}$ are the inclusion maps, this equation says that $\eta^{*}$ induces $\psi^{*}$.

(ii) Similar.

Lemma 2. (i) $A_{k}{ }^{*}=A^{* k}$.

(ii) $X^{k *}=X^{*}{ }_{k}$.

Proof. (i) $A_{k}{ }^{*}=G^{*} / A_{k}{ }^{0}=G^{*} /\left(A^{0}+\left(p^{k} G\right)^{0}\right)=A^{* k}$, since $\left(p^{k} G\right)^{0}=G^{*}\left[p^{k}\right]$.

(ii) Use (i) with $X^{*} \leq G^{*}$ in place of $A \leq G$ and then apply *.

Proof of Theorem. $(\mathrm{Z}) \Rightarrow(\mathrm{H})$. Assume that statement $(\mathrm{Z})$ holds. One implication in $(\mathrm{H})$ is trivial. For the other, suppose we are given an isomorphism $\psi: X \rightarrow Y$ that induces an isomorphism $X^{k} \rightarrow Y^{k}$ for each $k$. By Lemma 1(i) and Lemma 2(ii), the isomorphism $\psi^{*}: Y^{*} \rightarrow X^{*}$ induces an isomorphism $Y^{*}{ }_{k} \rightarrow X^{*}{ }_{k}$ for each $k$. Part (Z) of the theorem applies to give an isomorphism $\eta: H^{*} \rightarrow G^{*}$ that induces $\psi^{*}$. Using Lemma 1 (ii) and natural isomorphisms, we obtain an isomorphism $G \rightarrow H$ that induces $\psi$.

$(\mathrm{H}) \Rightarrow(\mathrm{Z})$. Similar. 


\section{REFERENCES}

1. P. Hill, An isomorphism theorem for group pairs of finite abelian groups, Publ. Math. Debrecen 43/3-4 (1993), 343-349.

2. I. Kaplansky, Infinite Abelian groups, Univ. of Michigan Press, Ann Arbor, MI, 1954.

3. L. Zippin, Countable torsion groups, Ann. of Math. 36 (1935), 86-99.

Department of Mathematics, Auburn University, Alabama 36849-5310

E-mail address: holmerromail . auburn.edu 